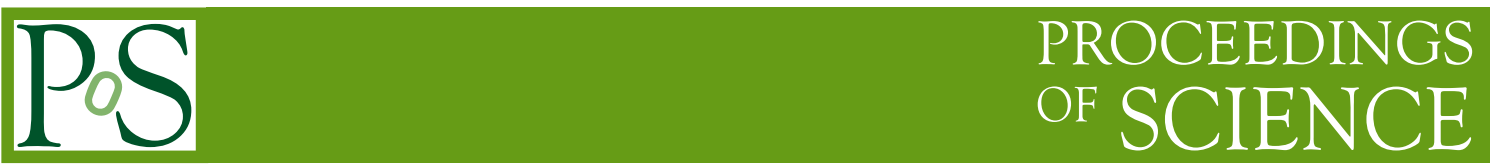

\title{
Neutron Spin Sum Rules and Spin Polarizabilities at Low $Q^{2}$
}

\author{
Vincent Sulkosky ${ }^{* \dagger}$ \\ Massachusetts Institute of Technology \\ E-mail: vasulk@jlab.org
}

Jefferson Lab has become one of the premier facilities for the study of nucleon spin structure. In the past decade, several high precision measurements have been performed that have extended our knowledge of the spin-dependent structure functions $g_{1}\left(x, Q^{2}\right)$ and $g_{2}\left(x, Q^{2}\right)$ between $Q^{2}=0.02-5 \mathrm{GeV}^{2}$. From the measured nucleon structure functions, spin moments and polarizabilities have been formed. These proceedings will concentrate on recent measurments on the neutron via a polarized ${ }^{3} \mathrm{He}$ target in the low $Q^{2}$ region. In particular, experiment E97-110 was performed in Hall A to study the neutron and ${ }^{3} \mathrm{He}$ spin structure at four-momentum transfers between 0.02 and $0.3 \mathrm{GeV}^{2}$. This $Q^{2}$ range allows us to make a benchmark-check of Chiral Perturbation Theory calculations. Previous results for the spin polarizabilities down to $0.1 \mathrm{GeV}^{2}$ have shown surprising disagreement with these calculations. Preliminary results on the first moments of the neutron spin structure functions are presented.

6th International Workshop on Chiral Dynamics, CD09

July 6-10, 2009

Bern, Switzerland

\footnotetext{
*Speaker.

${ }^{\dagger}$ On behalf of the Jefferson Lab E97-110 Collaboration
} 


\section{Introduction}

The study of nucleon spin structure has been an active field over the past few decades and has employed the use of sum rules. In particular, the Gerasimov-Drell-Hearn (GDH) [1] sum rule for real photon scattering $\left(Q^{2}=0\right)$. The expression for a spin- $\frac{1}{2}$ target is given by:

$$
\int_{v_{0}}^{\infty}\left[\sigma_{\frac{1}{2}}(v)-\sigma_{\frac{3}{2}}(v)\right] \frac{\mathrm{d} v}{v}=-\frac{2 \pi^{2} \alpha}{\mathrm{M}^{2}} \kappa^{2}
$$

where $\kappa$ and $\mathrm{M}$ are the anomalous magnetic moment and mass of the target, $v_{0}$ the inelastic threshold, $v$ the photon energy and $\alpha$ is the electromagnetic coupling constant. In this expression, $\sigma_{\frac{1}{2}}\left(\sigma_{\frac{3}{2}}\right)$ are the photoabsorption cross-sections where the photon helicity is parallel (anti-parallel) to the target spin. This sum rule indicates that there is a close relationship between the nucleon's excitation spectrum and its anomalous magnetic moment, which was one of the first pieces of evidence that the nucleon has an internal structure. The GDH sum rule is also valid for any target with spin- $S$.

In 1989, the GDH integral was first extended [2] to virtual photon absorption $\left(Q^{2}>0\right)$. Later other extensions were proposed $[3,4]$. One approach leads to the following equations:

$$
\begin{aligned}
I_{G D H}\left(Q^{2}\right) & =\int_{v_{0}}^{\infty}\left[\sigma_{\frac{1}{2}}\left(v, Q^{2}\right)-\sigma_{\frac{3}{2}}\left(v, Q^{2}\right)\right] \frac{d v}{v} \\
& =\frac{16 \pi^{2} \alpha}{Q^{2}} \int_{0}^{x_{0}}\left[g_{1}\left(x, Q^{2}\right)-\frac{4 M^{2} x^{2}}{Q^{2}} g_{2}\left(x, Q^{2}\right)\right] d x,
\end{aligned}
$$

where $x_{0}=\frac{Q^{2}}{2 M v_{0}}$, and $x$ is the Bjorken scaling variable. Here the photoabsorpton cross-sections have been replaced by the electroproduction cross-sections. Another extension [4] relates the integral to the forward virtual Compton scattering amplitudes $S_{1}\left(Q^{2}\right)$ and $S_{2}\left(Q^{2}\right)$ to form a $\mathrm{Q}^{2}$ dependent sum rule. This extension provides a relation that is constrained at the two ends of the $Q^{2}$ spectrum by well known sum rules: GDH at $Q^{2}=0$ and the Bjorken [5] sum rule at large $Q^{2}$. The extended GDH sum rule can be used to make comparisons between theoretical calculations and experimental data over the entire $Q^{2}$ range. The comparisons allow an investigation of the transition between the non-perturbative region and the perturbative region of Quantum Chromodynamics (QCD). Refs. [6, 7] provide detailed reviews on the experimental and theoretical efforts in this field.

Theoretical calculations from QCD are difficult to obtain due to the theory's non-perturbative nature in the low energy regime. In this region, moments of the spin structure functions have been calculated using Chiral Perturbation Theory (ChPT), which is a low-energy effective field theory. Experiment E97-110 [8] was specifically conducted to study the neutron spin structure functions at very low $Q^{2}$ and to make a benchmark check of ChPT calculations for these moments. In these proceedings, the data at low and intermediate $Q^{2}$ are reviewed. The experimental details and preliminary results of experiment E97-110 follow.

\section{Recent Results on Neutron Spin Structure}

Due to the recent technological advances in polarized beams and targets, experiments at Jefferson Lab have allowed precise measurements of the nucleon spin structure functions down to 
$Q^{2}=0.02 \mathrm{GeV}^{2}$. Hall A measurements have focused on the neutron [9], which are extracted from data on a polarized ${ }^{3} \mathrm{He}$ target; whereas, Hall B measurements [10] have involved polarized proton and deuteron targets. In these proceedings, only the neutron results from polarized ${ }^{3} \mathrm{He}$ experiments will be discussed.

Previous data and calculations for the extended GDH integral for the neutron are displayed in Fig. 1. At $Q^{2}=0$, the neutron prediction for the GDH sum rule is shown by the arrow $(\sim-233 \mu \mathrm{b})$. High- $Q^{2}$ results from HERMES [11] are shown by the solid squares. The open circles display the resonance contribution from the Hall A results [9], which are considerably more negative than the MAID resonance model [3]. The solid-inverted triangles include an estimate of the unmeasured low- $x$ contribution. The statistical uncertainties are displayed on the data points; whereas, the gray error band represents the systematic uncertainties. Two next-to-leading order ChPT calculations are shown: heavy baryon ChPT (HBChPT) [12] and relativistic baryon ChPT (RBChPT) [13]. The shaded band also includes resonance effects for the RBChPT calculation. This band is the result of the uncertainties on the parametrization of the $\Delta$-resonance and vector meson contributions. The

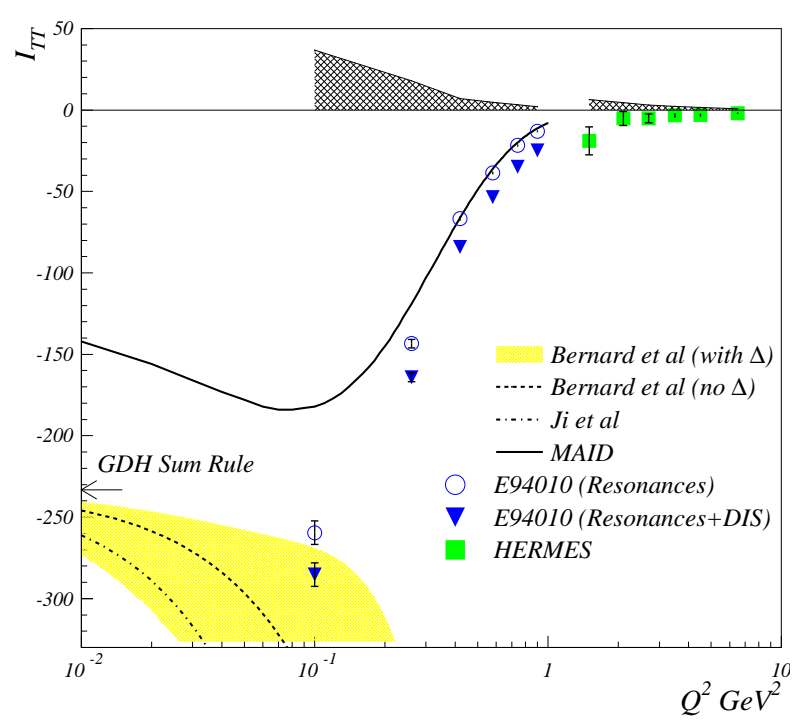

Figure 1: Neutron GDH integral $I\left(Q^{2}\right)$ results [9] shown with data from HERMES [11]. The solid curve indicates the MAID resonance model [3], and the dashed and dot-dashed curves represent the RBChPT [13] and HBChPT [12] calculations, respectively. The shaded band shows the relativistic calculation with the $\Delta$-resonance and vector meson contributions.

lowest $Q^{2}$ point $\left(0.1 \mathrm{GeV}^{2}\right)$, shows agreement with the RBChPT calculation with resonance effects. However the data set is right on the edge of where ChPT calculations are expected to be valid, and the calculation's uncertainty is large due to the parametrization of the resonances. Hence, data at lower $Q^{2}$ are required to make a definitive statement on these calculations.

The generalized spin polarizabilities involve higher moments in Bjorken- $x$ of the polarized structure functions. Due to the $x^{2}$ weighting, these integrals converge faster than the first moments, and therefore are significantly less sensitive to the unmeasured low- $x$ contributions. The expres- 
sions for the $\gamma_{0}$ and $\delta_{\mathrm{LT}}$ polarizabilites in terms of the structure functions are given by

$$
\begin{gathered}
\gamma_{0}=\frac{16 \alpha M^{2}}{Q^{6}} \int_{0}^{x_{0}} x^{2}\left[g_{1}\left(x, Q^{2}\right)-\frac{4 M^{2}}{Q^{2}} x^{2} g_{2}\left(x, Q^{2}\right)\right] d x \\
\delta_{\mathrm{LT}}=\frac{16 \alpha M^{2}}{Q^{6}} \int_{0}^{x_{0}} x^{2}\left[g_{1}\left(x, Q^{2}\right)+g_{2}\left(x, Q^{2}\right)\right] d x .
\end{gathered}
$$

The polarizability results for $\gamma_{0}$ and $\delta_{\mathrm{LT}}$ are shown in Fig. 2 top and bottom panels, respectively. For both plots, the next-to-leading order ChPT calculations are shown as the dashed (HBChPT) and dot-dashed (RBChPT) lines. The light shaded band also includes the $\Delta$ resonance and vector meson contributions for the RBChPT calculation. The MAID phenomenological model [3] is represented by the solid line. For $\gamma_{0}$, the RBChPT [13] calculation with resonance contributions is in good agreement with the experimental data at $0.1 \mathrm{GeV}^{2}$. However, the HBChPT [14] calculation shows a large discrepancy, which might indicate the importance of the resonances. Compared to MAID, the data is again significantly more negative for the lowest- $Q^{2}$ point. The $\delta_{\mathrm{LT}}$ spin polarizability

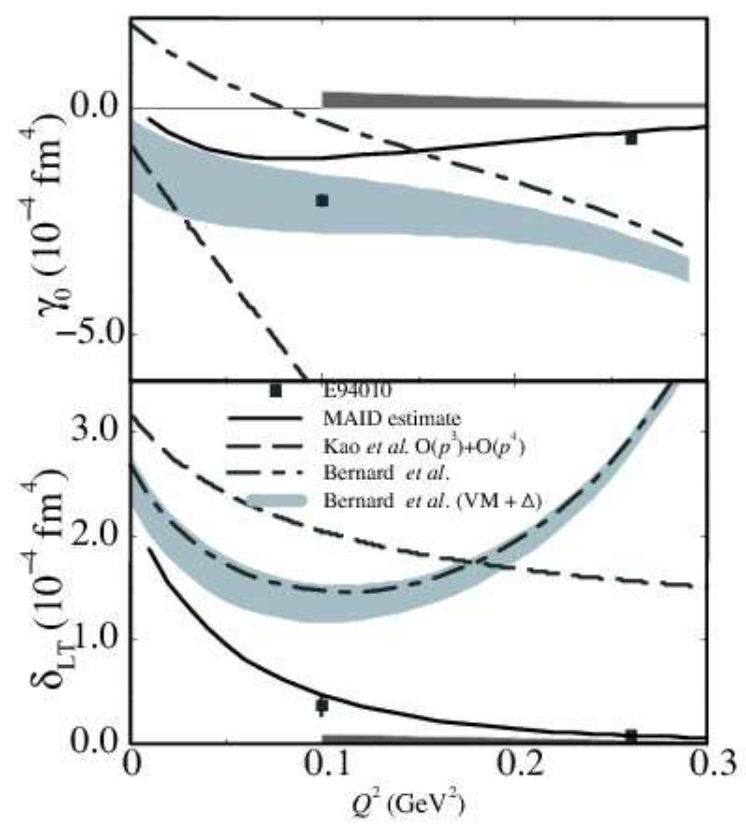

Figure 2: Spin polarizabilities $\gamma_{0}$ (top) and $\delta_{\mathrm{LT}}$ (bottom) on the neutron from the E94-010 experiment [9] shown with ChPT calculations of Ref. [13] (dot-dashed line) and Ref. [14] (dashed line) and the MAID model [3] shown by the solid line.

is expected to serve as a solid testing ground for ChPT, since it is insensitive to the $\Delta$ resonance contribution compared to $\gamma_{0}$. In the bottom panel of Fig 2 , the data for this quantity were also compared with the ChPT calculations and the MAID model. Here we see the surprising result that the data down to $0.1 \mathrm{GeV}^{2}$ show a large disagreement with both ChPT calculations. This disagreement has been referred to as the " $\delta_{\mathrm{LT}}$ puzzle" and presents a real challenge to ChPT theorists. One of the major limitations of the available data was that the lowest $Q^{2}$ might still be outside the region 
of ChPT's applicability. Additional experiments at Jefferson Lab have since obtained data for $Q^{2}$ down to $0.02 \mathrm{GeV}^{2}$.

\section{Recent Measurements and Preliminary Results}

Jefferson Lab experiment E97-110 acquired data in the Hall A [15] end station. A longitudinally polarized electron beam was scattered on either a longitudinally or transversely (in-plane) polarized ${ }^{3} \mathrm{He}$ target [15]. Polarized cross-section differences were measured for the inclusive reaction ${ }^{3} \mathrm{He}\left(\overrightarrow{\mathrm{e}}, \mathrm{e}^{\prime}\right)$. From these data, the polarized structure functions were extracted to form various moments at low $Q^{2}$ between 0.02 and $0.3 \mathrm{GeV}^{2}$. A comprehensive review of the experiment and data analysis can be found in Ref. [8]. Recently, the EG4 experiment [16] in Hall B obtained high precision data at similar $Q^{2}$ as experiment E97-110 for the proton and deuteron $g_{1}$ structure functions. During this conference, an update on the EG4 experiment and analysis was presented by S. Phillips.

After the structure functions have been extracted at constant $Q^{2}$, the extended GDH integral and other moments of the spin structure functions can be formed. The first moment of the $g_{1}$ structure function is given by

$$
\bar{\Gamma}_{1}\left(Q^{2}\right)=\int_{0}^{x_{0}} g_{1}\left(x, Q^{2}\right) d x .
$$

This integral is related to the GDH sum rule as $Q^{2} \rightarrow 0$ [17]:

$$
\lim _{Q^{2} \rightarrow 0} I_{G D H}\left(Q^{2}\right)=\frac{16 \pi^{2} \alpha}{Q^{2}} \bar{\Gamma}_{1}\left(Q^{2}\right) .
$$

In addition, the Burkhardt-Cottingham (BC) sum rule [18] is uniquely zero for any $Q^{2}$ and is related to the first moment of $g_{2}$ :

$$
\Gamma_{2}\left(Q^{2}\right)=\int_{0}^{1} g_{2}\left(x, Q^{2}\right) d x=0
$$

For this experiment, the polarized ${ }^{3} \mathrm{He}$ target was used as an effective neutron target. The neutron moments were extracted from the measured ${ }^{3} \mathrm{He}$ quantities by using the technique described in [19]. The systematic uncertainty due to this extraction was inferred to be $10 \%$ for $Q^{2} \leq 0.1 \mathrm{GeV}^{2}$ and $5 \%$ at higher $Q^{2}$. The deuteron data from EG4 will also provide an important systematic check of the neutron extraction from light nuclei. We have also utilized the parametrization in Ref. [20] to account for the unmeasured low- $x$ contribution.

In the left-hand side of Fig. 3, the preliminary results for the integral $\overline{\Gamma_{1}^{n}}$ are compared to theoretical calculations and earlier measurements $[9,10,11,21]$. The solid triangles show the preliminary data, and the inverted triangles indicate the results from a previous Hall A experiment [9] between $0.1 \mathrm{GeV}^{2}$ and $0.9 \mathrm{GeV}^{2}$. The error bars on the data indicate the size of the total uncertainties: both statistical and systematic. We expect to significantly improve the (largely dominant) systematic uncertainties for the final values. As $Q^{2}$ approaches zero, the data indicate a smooth transition and agree well with the earlier data and the two model calculations [22, 23]. Two calculations from Chiral Perturbation Theory $[12,13]$ are illustrated on the figure. The calculation with 

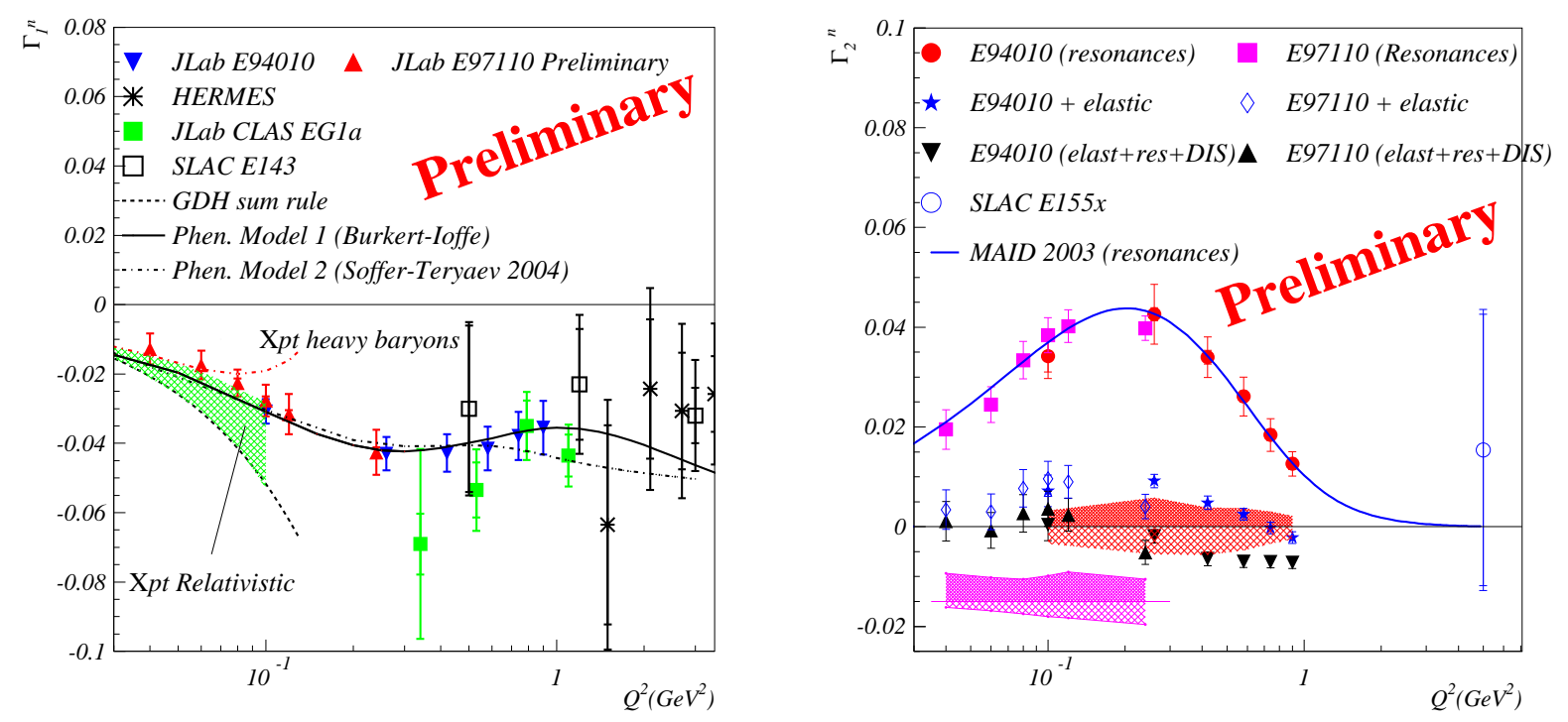

Figure 3: Preliminary neutron $\overline{\Gamma_{1}^{n}}$ (left) and $\mathrm{BC}$ sum, $\Gamma_{2}^{n}$, (right) results from experiment E97-110 [8] at very low $Q^{2}$. The $\Gamma_{2}^{n}$ integral is consistent with the $\mathrm{BC}$ sum rule prediction when all contributions are considered. For the left-hand side plot, the preliminary results (solid triangles) are plotted with earlier results from Jefferson Lab [9, 10], HERMES [11] and SLAC [21]. The ChPT calculations are also shown from Ji et al. [12] and Bernard et al. [13].

an estimate of the $\Delta$-resonance and vector meson contributions is represented by the shaded band. With the current uncertainties, the data show good agreement with the RBChPT calculation [13], and the three lowest $-Q^{2}$ points also agree well with the HBChPT calculation [12].

The integral $\Gamma_{2}^{n}$ is shown in the right-hand side of Fig. 3. The preliminary data agree well with the earlier neutron results [9], and the resonance data follow the MAID model [3]. The measured resonance data are displayed by the solid squares. After inclusion of the elastic contribution, the results are indicated by the open diamonds. The solid triangles represent the full integral with an estimate of the low- $x$ contribution; for this estimate we assumed $g_{2}=g_{2}^{W W}$, where $g_{2}^{W W}$ is the twist-2 part of $g_{2}$ derived by Wandzura and Wilczek [24]. The two bands indicate the systematic uncertainties; the darker band shows the total systematic uncertainty, and the lighter band shows an estimate of the systematic uncertainty for the unmeasured low- $x$ part. The total results are consistent with the BC sum rule expectation over the current measured $Q^{2}$ region. From the SLAC E155x collaboration [25], a high- $Q^{2}$ point (open circle) is also consistent with zero but with a large uncertainty.

The BC sum rule for the proton is largely unknown, since transversely-polarized target data are only available in a limited $Q^{2}$ range for the $g_{2}^{p}\left(x, Q^{2}\right)$ structure function. A new experiment [26] was recently approved to measure this structure function in the low $Q^{2}$ regime. Since $\delta_{\mathrm{LT}}^{p}$ also requires knowledge of $g_{2}^{p}\left(x, Q^{2}\right)$, this experiment combined with the EG4 $g_{1}^{p}$ data and the E97-110 neutron data will allow an isospin separation of the spin polarizabilities. 


\section{Summary}

Jefferson Lab experiment E97-110 was performed to provide precise spin structure data for the neutron and ${ }^{3} \mathrm{He}$ at low $Q^{2}$ between 0.02 and $0.3 \mathrm{GeV}^{2}$. From the measured structure functions, moments of the spin structure functions have been formed. At low $Q^{2}$, these quantities provide an important benchmark check of Chiral Perturbation Theory calculations. Neutron preliminary results for the first moments of the structure functions were shown and disc ussed. The first moment of $g_{1}^{n}$ has very good agreement with the higher- $Q^{2}$ data and the RBChPT calculation; on the other hand, only the three lowest $Q^{2}$ points agree with the HBChPT calculation. The integral $\Gamma_{2}^{n}$ is also consistent with the $\mathrm{BC}$ sum rule prediction. Final neutron results are expected after the systematic checks have been completed. In addition, the generalized forward spin polarizabilities are in the process of being extracted.

\section{Acknowledgments}

This work was supported by the U.S. Department of Energy contract DE-AC05-84ER40150, Modification No. M175, under which the Southeastern Universities Research Association operates the Thomas Jefferson National Accelerator Facility.

\section{References}

[1] S. B. Gerasimov, Sov. J. Nucl. Phys. 2 (1966) 430; S. D. Drell and A. C. Hearn, Phys. Rev. Lett. 16 (1966) 908.

[2] M. Anselmino, B. L. Ioffe and E. Leader, Sov. J. Nucl. Phys. 49 (1989) 136.

[3] D. Drechsel, S. S. Kamalov and L. Tiator, Phys. Rev. D 63 (2001) 114010 [hep-ph / 0008306 ];

D. Drechsel, B. Pasquini and M. Vanderhaeghen, Phys. Rept. 378 (2003) 99 [hep-ph/ 0212124 ].

[4] X. Ji and J. Osborne, J. Phys. G 27 (2001) 127 [hep-ph/9905410].

[5] J. D. Bjorken, Phys. Rev. 148 (1966) 1467; Phys. Rev. D1 (1970) 1376.

[6] J.-P. Chen, A. Deur and Z.-E. Meziani, Mod. Phys. Lett. A 20 (2005) 2745 [nucl-ex/ 0509007 ].

[7] S. E. Kuhn, J.-P. Chen and E. Leader, Prog. Part. Nucl. Phys. 63 (2009) 1 [hep-ph/08123535].

[8] V. Sulkosky, http://hallaweb.jlab.org/experiment/E97-110/thesis.html.

[9] M. Amarian et al., Phys. Rev. Lett. 89 (2002) 242301 [nucl-ex/ 020502 0];

ibid., Phys. Rev. Lett. 92 (2004) 022301 [hep-ex/ 0310003 ];

ibid., Phys. Rev. Lett. 93 (2004) 152301 [nucl-ex/ 0406005 ].

[10] R. Fatemi et al., Phys. Rev. Lett. 91 (2003) 222002 [nucl-ex/0306019];

J. Yun et al., Phys. Rev. C 67 (2003) 055204 [hep-ex/ 021204 4];

K.V. Dharmawardane et al., Phys. Lett. B 641 (2006) 11 [nucl-ex/ 0605028 ];

Y. Prok et al., Phys. Lett. B 672 (2009) 12 [nucl-ex/ 08022232 ].

[11] A. Airapetian et al., Eur. Phys. J. C 26 (2003) 527 [hep-ex/ 021004 7].

[12] X. Ji, C. Kao and J. Osborne, Phys. Lett. B 472 (2000) 1 [hep-ph/9910256]. 
[13] V. Bernard, T. R. Hemmert and U.-G. Meissner, Phys. Lett. B 545 (2002) 105 [hep-ph / 0203167$]$; ibid., Phys. Rev. D 67 (2003) 076008 [hep-ph/ 0212033$].$

[14] C. W. Kao, T. Spitzenberg and M. Vanderhaeghen, Phys. Rev. D 67 (2003) 016001 [hep-ph/0209241].

[15] J. Alcorn et al., Nucl. Instrum. Meth. A 522 (2004) 294.

[16] M. Battaglieri, A. Deur, R. De Vita and M. Ripani, JLab experiment E03-006.

[17] D. Drechsel and L. Tiator, Annu. Rev. Nucl. Part. Sci. 54 (2004) 69 [nucl-th/ 0406059 ].

[18] H. Burkhardt and W. N. Cottingham, Ann. Phys. 56 (1970) 453.

[19] C. Ciofi degli Atti and S. Scopetta, Phys. Lett. B 404 (1997) 223 [nucl-th/9606034].

[20] N. Bianchi and E. Thomas, Nucl. Phys. Proc. Suppl. 82 (2000) 256.

[21] P. L. Anthony et al., Phys. Rev. D 54 (1996) 6620 [hep-ex/9610007];

K. Abe et al., Phys. Rev. D 58 (1998) 112003 [hep-ph/9802357];

K. Abe et al., Phys. Rev. Lett. 79 (1997) 26 [hep-ex/9705012].

[22] V. D. Burkert and B. L. Ioffe, Phys. Lett. B 296 (1992) 223.

[23] J. Soffer and O. V. Teryaev, Phys. Rev. D 70 (2004) 116004 [hep-ph/ 0410228 ].

[24] S. Wandzura and F. Wilczek, Phys. Lett. B 72, (1977) 195.

[25] P. L. Anthony et al., Phys. Lett. B 553 (2003) 18 [hep-ex/ 0204028 ].

[26] A. Camsonne, J. P. Chen and K. Slifer, JLab experiment E08-027. 